\title{
Charge separation in organic photovoltaic cells
}

\author{
Paraskevas Giazitzidis ${ }^{\mathrm{a}}$, Panos Argyrakis ${ }^{\mathrm{a}}$, Juan Bisquert ${ }^{\mathrm{b}}$, Vyacheslav S. \\ Vikhrenko ${ }^{\mathrm{c}}$ \\ ${ }^{a}$ Department of Physics, University of Thessaloniki, Thessaloniki, Greece \\ ${ }^{b}$ Photovoltaics and Optoelectronic Devices Group, Departament de Fsica, Universitat \\ Jaume I, 12071 Castell, Spain \\ ${ }^{c}$ Belarusian State Technological University, Minsk, Belarus
}

\begin{abstract}
We consider a simple model for the geminate electron-hole separation process in organic photovoltaic cells, in order to illustrate the influence of dimensionality of conducting channels on the efficiency of the process. The Miller-Abrahams expression for the transition rates between nearest neighbor sites was used for simulating random walks of the electron in the Coulomb field of the hole. The non-equilibrium kinetic Monte Carlo simulation results qualitatively confirm the equilibrium estimations, although quantitatively the efficiency of the higher dimensional systems is less pronounced. The lifetime of the electron prior to recombination is approximately equal to the lifetime prior to dissociation. Their values indicate that electrons perform long stochastic walks before they are captured by the collector or recombined. The non-equilibrium free energy considerably differs from the equilibrium one. The efficiency of the separation process decreases with increasing the distance to the collector, and this decrease is considerably less pronounced for the three dimensional system. The simulation results are in good agreement with the extension of the continuum Onsager theory that accounts for the finite recombination rate at nonzero reaction radius and non-exponential kinetics of the charge separation process.
\end{abstract}

Keywords: charge separation, Electron-hole recombination, solar cells, dissociation kinetics, non-equilibrium Monte Carlo simulation, free energy

\section{Introduction}

Photovoltaic cells utilizing organic semiconductors have attracted much attention due to their promising electronic properties, low cost, thin film flexibility, and high functionality. Although their present efficiency is not high enough, widespread interest in both the academic and, increasingly, the commercial communities promises fast progress in this direction [1, 2]. Many attempts have been undertaken to understand the dissociation and recombination processes starting with the theoretical works of Onsager [3, 4, Frenkel [5] and Eigen [6], where models of these processes were suggested and investigated. Later, these models were refined with using proper boundary conditions for more adequate 
description of different stages of recombination/dissociation [7, 8, 9, 10, In these models it is assumed that continuum phenomenological diffusion equations are applicable on molecular space and time scales. On the other hand, discrete models were developed and their computer simulations were performed [11, 12, 13, 14, 15].

The models of charge separation were widely used to find routes for increasing the efficiency of the organic solar cells whose internal structure is characterized by a variety of characteristics, e. g. the charge transfer routs can be of different space dimensionality. For example, discotic liquid-crystal porphyrins conduct almost exclusively along one-dimensional backbones, most $\pi$-conjugated polymers have quasi-two-dimensional character, while $\mathrm{C}_{60}$ and their derivatives are truly three-dimensional organic semiconductors 16.

The effect of space dimensionality on recombination/dissociation was discussed earlier [17. It was shown that in the absence of interaction potential between two dissociating particles the escape probability (the probability that the particles can go away to infinity) is zero if the space dimension is smaller than or equal to two and increases with increasing space dimensionality.

Recently the entropy contribution with respect to the dimensionality of the organic semiconductor into charge separation after sunlight exciton production has been extensively discussed [1, 16. The equilibrium free energy of the electron-hole pair was used for estimating the charge separation efficiency in systems of different dimensionality. It was shown that the efficiency of three dimensional (3D) systems can be up to four orders of magnitude higher, as compared to an one dimensional (1D) system.

However, the equilibrium consideration does not take into account important features of the charge separation process as it was mentioned in Ref. [16] and is in more detail discussed below. Thus, we suggest a simple non-equilibrium model of geminate recombination/dissociation of electron-hole pairs and quantitatively investigate the electron yield on the collector depending on the system dimensionality with accounting of interparticle Coulomb interaction. The main focus of our work is the influence of the space dimensionality on the electron yield in zero electric field. The simulation results are compared with the extension of the Onsager continuum model [9, 10]. Characteristic times of the processes are considered as well.

\section{Model description}

To make the model as simple as possible we consider an electron that moves in a regular lattice and in the Coulomb field of the immobile hole [1, 16. Then, the electron can be considered as moving in the external Coulomb field of the hole and in the approximation of uniformly distributed lattice sites, its equilibrium distribution function can be written as

$$
f(r)=Q^{-1} n_{r} \exp [-\beta u(r)]=Q^{-1} \exp \left[-\beta\left[u(r)-k_{B} T S(r)\right]\right],
$$

where $Q$ is a normalization constant; $n_{r}=1,2 \pi r, 4 \pi r^{2}$ for one, two and three dimensional systems, respectively, and it determines the density of the number 
of lattice sites as a function of $r$ that can be occupied by the electron with equal probability; $r$ is the electron-hole distance; $T$ is the absolute temperature; $k_{B}$ Boltzmann constant; $\beta=1 /\left(k_{B} T\right)$ is the inverse temperature. The energy of Coulomb interaction for the electron on a cubic lattice with the lattice parameter $a$ is inversely proportional to electron-hole distance

$$
u(r)=\frac{-e_{0}}{\sqrt{i^{2}+j^{2}+k^{2}}}, e_{0}=\frac{e^{2}}{4 \pi \varepsilon_{0} \varepsilon a},
$$

where $\varepsilon$ is the medium dielectric constant; $e$ electron charge; $\varepsilon_{0}$ electric constant; $i, j, k$ are integers that determine lattice sites positions. For $1 \mathrm{D}$ and $2 \mathrm{D}$ systems the expression for the interaction energy has to be modified correspondingly. The second part of Eq. (1) is rewritten in such a way that the configuration entropy $S(r)=\ln n_{r}$ appears in a natural way. The expression in the square brackets determines the Helmholtz free energy

$$
\Delta G(r)=u(r)-k_{B} T S(r)
$$

that cumulatively takes into account the equal probability of occupying a number of equivalent lattice sites (with the same distance $r$ ) and the attractive electron-hole interaction that makes smaller $r$ distances much more preferable. Of course, this expression for $\Delta G$ is the same as given in [1, 16]. The distribution function is normalized to unity and the normalization constant

$$
Q=\sum_{i, j, k} \exp \left(-u(i, j, k) / k_{B} T\right)
$$

where the sum runs over all sites between the source and the collector.

We used kinetic Monte Carlo method with the Metropolis algorithm [18] to perform simulations of the equilibrium distribution functions. The parameters were taken from paper [16]: $\varepsilon=4, a=1 \mathrm{~nm}, T=300 \mathrm{~K}$. Then, the characteristic energy $e_{0} / k_{B} T \cong 13.9$ and the normalization constants are $Q \cong$ $(1.0882 ; 4.4448 ; 6.8888) \cdot 10^{6}$ for $1 \mathrm{D}, 2 \mathrm{D}$ and $3 \mathrm{D}$ cases, respectively. In the equilibrium simulation the electron can jump from the initial site to any other site inside of the collector $\left(i^{2}+j^{2}+k^{2} \leqslant N^{2}+N\right)$ except zero site $\left(i^{2}+j^{2}+k^{2}=0\right.$ where the hole is situated). The lattice sites are prescribed to the distance $r$ if (in 3D case with the corresponding changes in 2D and 1D cases) $r^{2}-r<$ $i^{2}+j^{2}+k^{2} \leqslant r^{2}+r, i, j, k, r$ and $N$ are integers and the collector is on the distance $(N+0.5) a$ from the hole. The configuration entropy in Monte Carlo simulation is taken into account indirectly through the interrogation of the lattice sites.

The electron energy difference $\Delta u$ between the destination and the initial state was used for calculating the probability of the electron transition between these two sites. The transition probability was taken equal to $\exp \left(-\Delta u / k_{B} T\right)$ if $\Delta u>0$ or 1 if $\Delta u \leqslant 0$. The free energy was calculated from the probabilities for the electron to occupy the lattice sites in accordance with Eq, 1

$$
\Delta G(r)=-k_{B} T\left[\ln f(r)+\ln Q-\ln n_{r}\right] .
$$


Definitely, in a true geminate recombination/dissociation process the electron cannot be in equilibrium with the hole. However, if the recombination rate is very small and can be neglected then the equilibrium model can be used for crude estimation of the electron spatial distribution around the hole.

\section{Results and discussion}

\subsection{Equilibrium results}

The results are shown in Fig $1 \mathrm{a}$ for $N=30$ (for the sake of uniformity in distribution of the lattice sites over distance $r$, the distance to the collector was taken equal to $30.5 a$ ) and they confirm the equilibrium distribution function (1) and the conclusion of papers [1, 16] about higher efficiency of 3D systems. Small fluctuations of the Monte Carlo simulation results around the analytical curves are explained by nonhomogeneous distribution of lattice sites over $r$ distances. The efficiency of charge separation in the equilibrium consideration was defined [1, 16] as the ratio of the probabilities for the electron to be on a certain distance $R$ from the hole and at its ground state that for Boltzmann statistics is proportional to $\exp \left(-\Delta E / k_{B} T\right)$, where $\Delta E$ is the free energy difference between these electron positions.

In Fig $1 \mathrm{Bb}$ the results for the systems with the distance of $500 a$ from the source to the collector are shown. It is evident that the efficiency of the systems estimated from the equilibrium free energy strongly depends on the distance between the source and the collector. The longer is this distance, the larger is the difference by many orders of magnitude in the efficiency of the systems of different dimensionality. Moreover, for $2 \mathrm{D}$ and $3 \mathrm{D}$ systems the longer is the distance from the source to the collector, the larger are the absolute values of the charge separation efficiency. This is counterintuitive to the real situation.

\subsection{Nonequilibrium results for the electron yield at the collector}

However, the equilibrium results do not take into account the finite lifetime of the electron before it arrives at the collector or recombines with the hole. Thus, non-equilibrium simulations are in order. Such simulations have been published earlier [12, 13, 14, however they were mainly focused on the electric field dependence of the electron yield. Analytical results are available as well [17. and they indicate that the larger the dimensionality is the larger survival probability for the electron exists at all distances. Moreover, $D=2$ is the critical dimensionality below which the electron-hole pair cannot dissociate (in the sense that the electron-hole distance tends to infinity) at non-zero recombination rate in the absence of the external field.

We have chosen as starting point of the electron the site close to the hole (on $1 \mathrm{~nm}$ distance) and $10^{6}-10^{7}$ electron trajectories were considered for each particular simulation to ensure good statistics. The simulations were performed for the system described above. The hole was fixed at the coordinate origin and the electron collector was taken at the distance of $30.5 \mathrm{a}$. Initially the electron was placed on the nearest neighbor to the origin's site. The transition rate 
for the electron to recombine from this initial site is designated as $w_{10}$. The transition rate of the electron to its other nearest neighbor site was calculated in accordance to Miller - Abrahams [19] expression

$$
\begin{gathered}
w_{n \rightarrow n+1}=w_{0} \exp \left[-\left(u_{n+1}-u_{n}+\left|u_{n+1}-u_{n}\right|\right) / 2 k_{B} T\right], \\
w_{0}=v_{0} \exp (-2 a / \alpha),
\end{gathered}
$$

where $u_{n}$ and $u_{n+1}$ are the electron energies at the initial and destination sites, respectively; $\alpha$ is the electron localization parameter; $w_{0}$ the hopping attempt frequency; $w_{0}$ determines the time scale of the charge separation process and the simulations were performed at $w_{0}=1$; then all the other rates are given in units of $w_{0}$. Thus, our non-equilibrium model corresponds to the discrete version of the Onsager recombination model 4 with accounting of finite time and non-zero reaction radius of geminate recombination according to [7, 8, 9] at zero electric field.

Although we consider the hole fixed at the origin, the results can be used for situations when both charges are moving with the proper definition of the diffusion constants [8, 10]. On the other hand, the simulation results are valid for the system with separated electron subspace by reflecting boundary conditions for the plane passing through the immovable hole. This means that if the electron occupies site $i=0,|j|+|k| \neq 0$ and the trail is $i=-1$ then it has to be taken $i=1$.

We can consider three possibilities: $(a)$ the electron of the just created exciton can immediately or after some waiting time recombine; $(b)$ the electron can recombine after random walks over the lattice at the influence of a quite strong Coulomb electron-hole interaction; $(c)$ the electron can reach the collector (dissociation). The characteristic time of process $(a)$ is estimated as $w_{10}^{-1}$. However, the total recombination rate is determined by both $(a)$ and $(b)$ processes. The distribution of the recombination and the electron arrival at the collector times can easily be extracted from Monte Carlo simulation results.

The simulation results are shown in the Table and, for visibility, in Fig $2 \mathrm{a}$. The recombination rates (the electron transition to the origin in process $(a)$ ) are shown in the first column. For comparison, the dimensionless transition rate for an electron from the nearest to the origin site to its second neighbor in $1 \mathrm{D}$ system is equal to $w_{12}=\exp (-13.9 / 2) \cong 0.00096$, while in $2 \mathrm{D}$ and $3 \mathrm{D}$ systems it is considerably larger, $w_{13}=\exp \left(-13.9\left(1-2^{-1 / 2}\right)\right) \cong 0.017$; the transition rates grow quickly with increasing the electron-hole distance. The next three columns in the Table contain the electron yields at the collector (the ratios of the number of electrons reached the collector to the total number of electrons for each particular value of $w_{10}$ ) in $1 \mathrm{D}, 2 \mathrm{D}$ and $3 \mathrm{D}$ systems, respectively. The three last columns contain the ratios of the electron yields for the systems shown in the column headers. These ratios are almost constant in wide range of the recombination rates up to the point where the yield achieves values larger than approximately 0.1 . Some fluctuations in the last column are due to poor statistics of 1D systems at low electron yields. 
Table 1: The electron yields and their ratios for systems of different dimensionality.

\begin{tabular}{lccccccr}
$w_{10}$ & $1 \mathrm{D}$ & $2 \mathrm{D}$ & $3 \mathrm{D}$ & $3 \mathrm{D}(\mathrm{Eq} .9)$ & $2 \mathrm{D} / 1 \mathrm{D}$ & $3 \mathrm{D} / 2 \mathrm{D}$ & $3 \mathrm{D} / 1 \mathrm{D}$ \\
\hline 0.01 & $7.00 \cdot 10^{-6}$ & $2.23 \cdot 10^{-4}$ & $3.85 \cdot 10^{-3}$ & $3.85 \cdot 10^{-3}$ & 31.8 & 17.3 & 550 \\
0.005 & $1.27 \cdot 10^{-5}$ & $4.40 \cdot 10^{-4}$ & $7.65 \cdot 10^{-3}$ & $7.67 \cdot 10^{-3}$ & 34.6 & 17.4 & 602 \\
0.002 & $3.45 \cdot 10^{-5}$ & $1.13 \cdot 10^{-3}$ & $1.89 \cdot 10^{-2}$ & $1.90 \cdot 10^{-2}$ & 32.8 & 16.7 & 548 \\
0.001 & $6.77 \cdot 10^{-5}$ & $2.25 \cdot 10^{-3}$ & $3.70 \cdot 10^{-2}$ & $3.72 \cdot 10^{-2}$ & 33.2 & 16.4 & 547 \\
0.0005 & $1.28 \cdot 10^{-4}$ & $4.49 \cdot 10^{-3}$ & $7.17 \cdot 10^{-2}$ & $7.17 \cdot 10^{-2}$ & 35.1 & 16 & 560 \\
0.0003 & $2.22 \cdot 10^{-4}$ & $7.45 \cdot 10^{-3}$ & 0.114 & 0.114 & 33.6 & 15.3 & 514 \\
0.0001 & $6.57 \cdot 10^{-4}$ & $2.20 \cdot 10^{-2}$ & 0.279 & 0.278 & 33.6 & 12.7 & 425 \\
0.00005 & $1.33 \cdot 10^{-3}$ & $4.32 \cdot 10^{-2}$ & 0.436 & 0.436 & 32.5 & 10.1 & 328 \\
0.00001 & $6.62 \cdot 10^{-3}$ & 0.184 & 0.794 & 0.794 & 27.8 & 4.3 & 120
\end{tabular}

Thus, it is evident that the competition of the configuration entropy contribution and Coulomb interaction gives rise to higher efficiency of higher dimensional systems in the charge recombination/dissociation processes.

Referring to the ergodicity hypothesis it is possible to say that in equilibrium the electron is moving on the equilibrium free energy surface and at the same time this surface determine according to Eq.(1) the probability distribution of the equilibrium ensemble of electrons over the lattice sites. Thus, we can consider as a mathematical construction the non-equilibrium free energy calculated by

$$
\Delta G(r)=-(\ln Q+\ln f(r)) k_{B} T,
$$

where $f(r)$ is the non-equilibrium distribution function evaluated from the Monte Carlo simulation results and normalized to unity. On the other hand, the Monte Carlo simulation procedure can be considered as reproducing the electron motion over this non-equilibrium free energy surface.

The equilibrium and non-equilibrium free energies can be used for comparing the distribution of times spent by the electron on lattice sites because the distribution functions themselves vary by many orders of magnitude and are not convenient for comparison. The non-equilibrium free energy (Fig 3 ) considerably differs from the equilibrium one. Its prominent feature is that it does not depend on the recombination rate. This energy strongly increases near the collector that indicates significant decrease of probability distribution due to absorption of the electrons by the collector.

\subsection{Comparison with the continuum representation}

The current simulation results can be compared with the extension [9, 10] of the continuum Onsager model that takes into account the finite geminate recombination rate at nonzero reaction radius and the non-exponential character of the electron-hole separation process. As we calculated the electron yield $Y(q a / a)$ on a certain distance ( $q a)$ from the hole, starting from the distance $a$ it is possible to extract the corresponding value from the expression [9, 10] for 


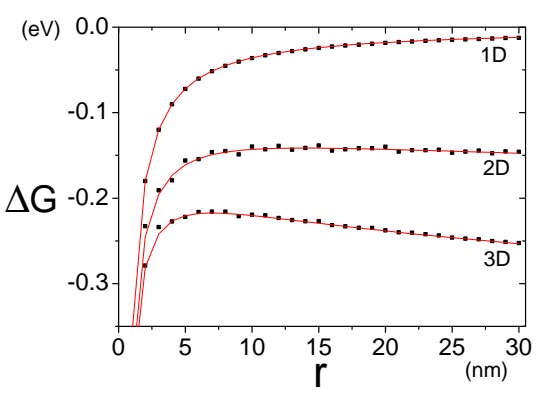

(a)

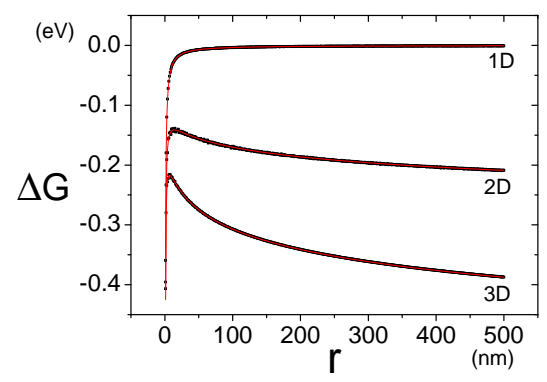

(b)

Figure 1: (color online) The free energy versus the electron-hole distance for one, two, and three dimensional systems of size (a) 30a; (b) 500a. The lines reproduce the analytic expressions of $\mathrm{Eq} 3$, the dots are the Monte Carlo simulation results.

the yield at infinite distance when starting from the distances $a(Y(\infty / a))$ and $q a(Y(\infty / q a))$ using the probability product rule

$$
Y(q a / a)=Y(\infty / a) / Y(\infty / q a)=\left[1+\frac{p a^{2}}{D r_{c}}\left(\exp \left(\frac{q-1}{q} \frac{r_{c}}{a}\right)\right)\right]^{-1},
$$

where $q=N+0.5, p$ is a reactivity parameter, $D$ the diffusion coefficient, $r_{c}=e^{2} /\left(4 \pi \varepsilon_{0} \varepsilon k_{B} T\right)$ the Onsager radius; in our simulation $\left(r_{c} / a\right)=13.9$ and $D=a^{2}$ because the frequency factor was taken equal to 1 .

The main problem in comparing our discrete model results with the continuum theory [9, 10] is the evaluation of the reactivity parameter $p$. Comparing to the $3 \mathrm{D}$ case, the recombination rate as the flux through the spherical surface around the hole 10 and the flux from the volume $6 a^{3}$ where recombination occurs we arrive at the estimation $p=(3 / 2 \pi) w_{10} a \cong 0.477 w_{10} a$ and then $p a^{2} / D r_{c} \cong 0.0343 w_{10}$. However, with this value of $p$ the theoretical results were systematically higher by some $10 \%$ of the simulation results for $N=30$. Thus, slightly larger values $p=0.522 w_{10} a$ were used and the calculation results are given in the fifth column of the table. As long as the second term in Eq9 


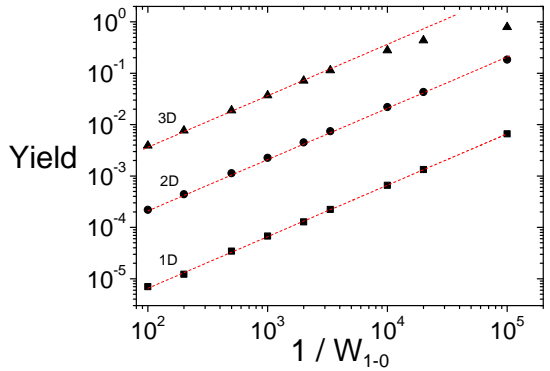

(a)

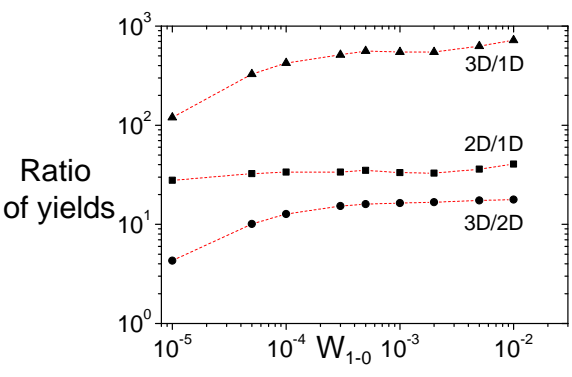

(b)

Figure 2: (Color online) The electron yields (a) and their ratios (b) versus recombination rates for the systems of different dimensionality. Straight dashed lines are the linear fittings, while curves are guides for the eyes.

is around 10 or larger the yield is inversely proportional to the recombination rate $w_{10}$. The non-equilibrium simulation results qualitatively confirm the equilibrium estimations, although quantitatively the higher efficiency of the higher dimensional systems is less pronounced and depends on the ratio of the recombination and the transition rates in the vicinity of the hole. In 1D system the yield is almost exactly inversely proportional to the recombination rate, while in 2D and 3D systems the saturation effect causes deviations from such behavior at high values of the yield.

As it was mentioned above (Fig $1 \mathrm{~b}$ ) the equilibrium consideration predicts an increase of the charge separation efficiency in the 2D and 3D systems with increasing the distance to the collector, something that is counterintuitive because it does not take into account the kinetics of the process. The non-equilibrium simulations for a larger system of $N=50$ at $w_{10}=0.0003$ have shown that the absolute values of the electron yields are equal to $9.48 \cdot 10^{-5}, 4.8 \cdot 10^{-3}$, and $9.78 \cdot 10^{-2}$ for $1 \mathrm{D}, 2 \mathrm{D}$, and $3 \mathrm{D}$ systems, respectively, and they are smaller than the corresponding values for the system of $N=30$ by $2.27,1.55$, and 1.16 times. This means that although the absolute values of the yields decrease with increasing distance to the collector the relative efficiency of higher dimensional systems increases. Of course, 30 or $50 \mathrm{~nm}$ are too small distances as compared to that electrons have to move in modern photovoltaic devices. Nevertheless, these distances correspond respectively to more than 2 and almost 4 Onsager radiuses and thus capture the main features of the dissociation/recombination process.

To adjust the theoretical value of $\mathrm{Eq} 9$ for $N=50$ with the simulation result it is necessary to take $p=0.514 w_{10} a$ which is smaller than that for $N=30$ and closer to the theoretical value $p=0.477 w_{10} a$, because at larger scale the discrepancy between discrete and continuum approaches becomes less pronounced. Moreover, separation of charges is an initial value problem and thus memory effects contribute to diffusion on a lattice 20. even when only 
one particle is considered [21] while they are not taken into account by the continuum diffusion equation used in the Onsager and other such models. The difference in $10 \%$ only between the results of the continuum and discrete models is surprising in view of application of continuum representations up to molecular scales.

It is interesting to note that the geminate charge recombination in the $3 \mathrm{D}$ heterojunction system with planar interlayer boundary can be formally described by the system of differential equations in four dimensions [22]. On this basis the increase of the escape probability in systems with heterojunction following from the dynamic Monte Carlo simulation [13, 15] was explained [22]. Really, if we consider for simplicity a one-dimensional heterojunction systems then for a given electron-hole distance in $r$ lattice spacings there are $r$ energetically equivalent positions that result in exactly the same equilibrium distribution function as for the 2D system without heterojunction. However, the situation is to some extent controversial. In heterojunction systems the total configuration entropy of the system decreases because of braking its translational symmetry. From physical point of view it means that in systems without heterojunction excitons can be created in any space position, while in heterojunction systems the excitons have to be created at the interlayer boundary or the excitons have to be efficiently transported to this boundary prior to their recombination.

The theoretical result [9, 10, can be easily used for investigating how the type of the lattice influences the electron yield. For example, for the face centered cubic lattice for the same nearest neighbor distance $a$ the recombination volume and the diffusion coefficient 23 are equal to $6 \sqrt{2} a^{3}$ and $2 a^{2}$, respectively. Thus, the coefficient $\left(p a^{2} / D r_{c}\right)$ is smaller by a factor of $\sqrt{2}$ than the case of the simple cubic lattice, which in turn results in grater electron yield. Moreover, in this case the density of lattice sites is larger by the $\sqrt{2}$ factor as well. For equal densities the nearest neighbor distance has to be $\sqrt[6]{2}$ times larger, which will result in strong increase (approximately by a factor of 4 at accepted conditions) of the electron yield in the face centered cubic lattice as compared to the simple cubic lattice. The influence of the type of the lattice on the recombination/separation process was considered in Ref.[24] in another context in the model involving more complicated parameterization.

\subsection{The electron lifetimes}

The distribution of electron lifetimes before arriving at the collector is shown in Fig 4. The total number of electrons was $10^{7}$, however only a part of them indicated in the Table arrived at the collector. To make the results more transparent the total simulation time was split in bins of 50 Monte Carlo steps (MCs) and the electrons were collected in each bin. A small number of electrons went quickly to the collector while the majority arrived at the collector after long stochastic walks over the lattice. Thus, the distributions of arrival times have maximal values at several thousand of MCs. The decaying parts of the curves were fitted by exponentially decaying functions and the relaxation times are inversely proportional to the recombination rates as shown in the insert of Fig, 4 


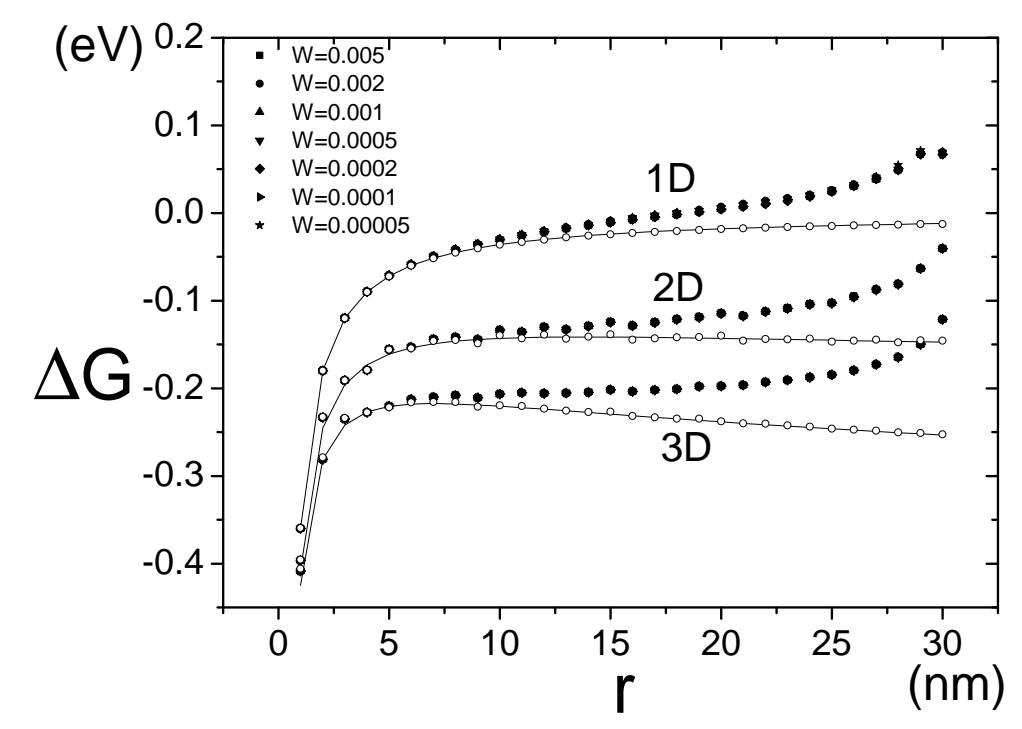

Figure 3: The nonequilibrium free energy (full symbols) versus electron-hole distance for different recombination rates. Note that all full symbols contain seven (7) sets of data which all fall on the top of each other in each case (i.e the full symbols contain 21 sets of data). Open circles are for the equilibrium Monte Carlo simulation results, the curves represent the analytical expression (3) for $\Delta G$.

they increase with the lattice dimensionality because the larger the dimensionality the longer electron walks are in order. The same dependence is observed for the distribution of the recombination times as is shown in Fig 5 , except that this distribution decays from the very beginning. It is worth to note that the relaxation times are approximately the same for the distributions of arrival and recombination times. For comparison, we can calculate the portion of electrons that recombine according to process (a) as $w_{10} /\left(w_{10}+w_{12}\right)$ for $1 \mathrm{D}$ system and as $w_{10} /\left(w_{10}+w_{12}+2 w_{13}\right)$ for $2 \mathrm{D}$ system. For the $3 \mathrm{D}$ system a multiplication by 4 in the last expression must be used instead of 2 . For the system with $w_{10}=0.0003$ we get $0.238,0.0164$ and 0.0085 for $1 \mathrm{D}, 2 \mathrm{D}$ and $3 \mathrm{D}$ cases, respectively. Thus, in 1D system a significant part of the electrons recombine immediately after the exciton creation and the details of random walks in the vicinity of the hole that depend on the structure of the lattice strongly influence the electron yield. The recombination time of the process (a) $w_{10}^{-1}=3333 \mathrm{MCs}$ is the same for all dimensionalities, while for (a) and (b) processes in accordance with Fig 5 they are 7000, 13500, and 18500 MCs for 1D, 2D and 3D systems, correspondingly.

Fig 4 demonstrates that the electron-hole separation process is strongly non- 
exponential during the initial period which is considerably shorter than the subsequent exponential decay. Thus, although the criticism [10] of the OnsagerEigen-Braun type of models is reasonable, the influence of this non-exponential character of the separation process can be of minor importance for recombination/dissociation, and these models can be used for analyzing experimental results.

\section{Conclusion}

Summarizing, the non-equilibrium simulation results show that the efficiency of charge separation in 3D systems is more than an order of magnitude larger, as compared to $2 \mathrm{D}$ systems and almost three orders of magnitude larger than that in 1D systems at comparable conditions. The ratio of efficiencies does not depend on the recombination rate if the electron yield is lower than 0.1. Surprisingly, the lifetimes of the collected and recombined electrons are approximately equal and their values indicate that electrons perform long stochastic walks before they are captured by the collector or recombined. This phenomenon is explained by the fact that only a small amount of electrons reaches the collector and those ones that are able to move far from the origin can arrive at the collector or recombine with comparable probabilities. Just these electrons determine the relaxation times. The simulation results compare well with the predictions of the continuum theory [9, 10] that accounts for the finite recombination rate at nonzero reaction radius and non-exponential kinetics of the charge separation process, if the reaction volume is taken equal to the total volume of primitive cells that are the nearest neighbors of the cell where the hole is situated. This agreement validates the correspondence of the continuum and discrete models as well as the simulation results.

\section{References}

[1] T. Clarke, J. Durrant, Charge photogeneration in organic solar cells, Chem. Rev. 110 (2010) 6736-6767.

[2] C. Deibel, V. Dyakonov, Polymer-fullerene bulk heterojunction solar cells, Rep. Progr. Phys. 73 (2010) 096401.

[3] L. Onsager, Deviations from Ohm's law in weak electrolytes, The Journal of Chemical Physics 2 (1934) 599-615.

[4] L. Onsager, Initial recombination of ions, Phys. Rev. 54 (1938) 554-557.

[5] J. Frenkel, On pre-breakdown phenomena in insulators and electronic semiconductors, Phys. Rev. 54 (1938) 647-648.

[6] M. Eigen, The thermodynamics of electrolytes at higher concentration, Phys. Chem. 58 (1954) 702-714. 
[7] J. Noolandi, K. Hong, Solution of the Smoluchowski equation with a Coulomb potential. I. General results, The Journal of Chemical Physics 68 (1978) 5163-5171.

[8] J. Noolandi, K. M. Hong, Theory of photogeneration and fluorescence quenching, The Journal of Chemical Physics 70 (1979) 3230-3236.

[9] H. Sano, M. Tachiya, Partially diffusion-controlled recombination, The Journal of Chemical Physics 71 (1979) 1276-1282.

[10] M. Wojcik, M. Tachiya, Accuracies of the empirical theories of the escape probability based on Eigen model and Braun model compared with the exact extension of Onsager theory, The Journal of Chemical Physics 130 (2009) 104107.

[11] H. Scher, S. Rackovsky, Theory of geminate recombination on a lattice, The Journal of Chemical Physics 81 (1984) 1994-2009.

[12] U. Albrecht, H. Bssler, Yield of geminate pair dissociation in an energetically random hopping system, Chem. Phys. Lett. 235 (1995) 389-393.

[13] P. Peumans, S. R. Forrest, Separation of geminate charge-pairs at donoracceptor interfaces in disordered solids, Chem. Phys. Lett. 398 (2004) 2731.

[14] C. Deibel, T. Strobel, V. Dyakonov, Origin of the efficient polaron-pair dissociation in polymer-fullerene blends, Phys. Rev. Lett. 103 (2009) 036402.

[15] M. Wojcik, P. Michalak, M. Tachiya, Geminate electron-hole recombination in organic solids in the presence of a donor-acceptor heterojunction, Applied Physics Letters 96 (2010).

[16] B. A. Gregg, Entropy of charge separation in organic photovoltaic cells: The benefit of higher dimensionality, Journ. Phys. Chem. Lett. 2 (2011) 3013-3015.

[17] A. V. Barzykin, M. Tachiya, Diffusion influenced reaction kinetics on fractal structures, The Journal of Chemical Physics 99 (1993) 9591-9597.

[18] N. Metropolis, A. W. Rosenbluth, M. N. Rosenbluth, A. Teller, E. Teller, Equation of state calculations by fast computing machines, The Journal of Chemical Physics 21 (1953) 1087-1092.

[19] A. Miller, E. Abrahams, Impurity conduction at low concentrations, Phys. Rev. 120 (1960) 745-755.

[20] L. Skarpalezos, N. Tsakiris, P. Argyrakis, V. S. Vikhrenko, Memory effects in strongly interacting lattice gases: Self-intermediate scattering function studies, Phys. Rev. B 84 (2011) 075476. 
[21] S. Chandrasekhar, Stochastic problems in physics and astronomy, Rev. Mod. Phys. 15 (1943) 1-89.

[22] M. Wojcik, M. Tachiya, Long-time decay kinetics of geminate electron-hole pairs in donor-acceptor heterojunction systems, Chemical Physics Letters 537 (2012) 58-61.

[23] J. Manning, Diffusion kinetics for atoms in crystals, Van Nostrand, 1968.

[24] S. Rackovsky, H. Scher, Effect of lattice topology on geminate recombination, Chem. Phys. Lett. 117 (1985) 271-273. 


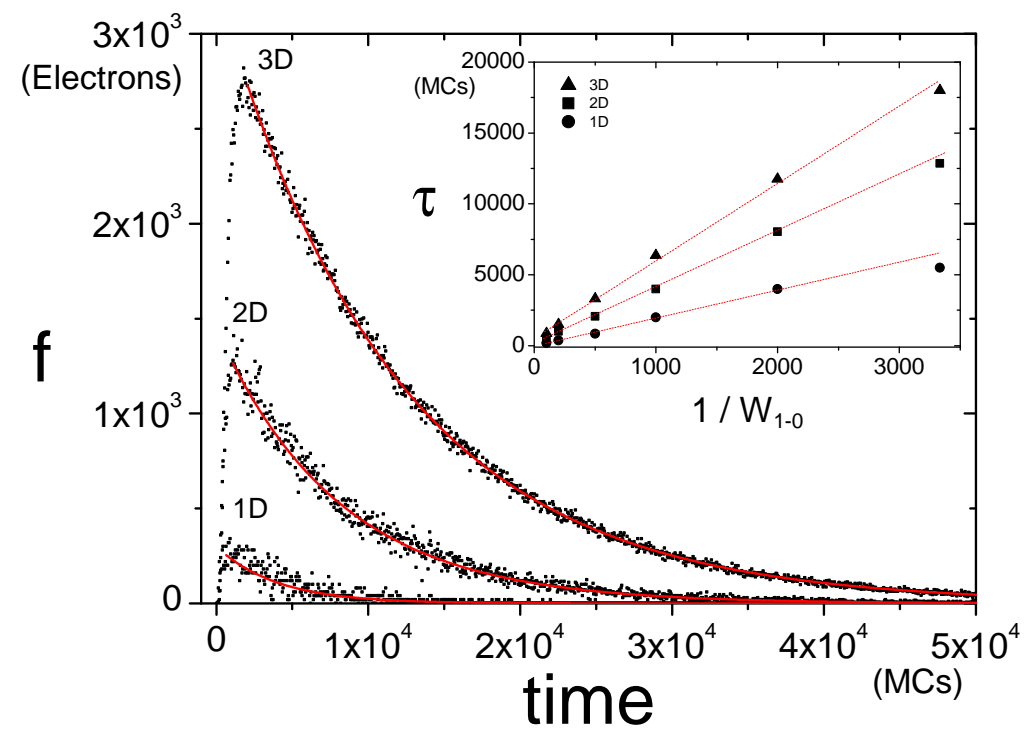

Figure 4: (color online)The distribution of electron lifetimes before arriving at the collector for $1 \mathrm{D}, 2 \mathrm{D}$ and $3 \mathrm{D}$ systems for $w_{10}=0.0005$. The frequencies for $1 \mathrm{D}$ and $2 \mathrm{D}$ systems are multiplied by 10 and 5 , respectively. In the insert: the relaxation times of the arrival times distributions of the collected electrons versus the inverse recombination rates. 


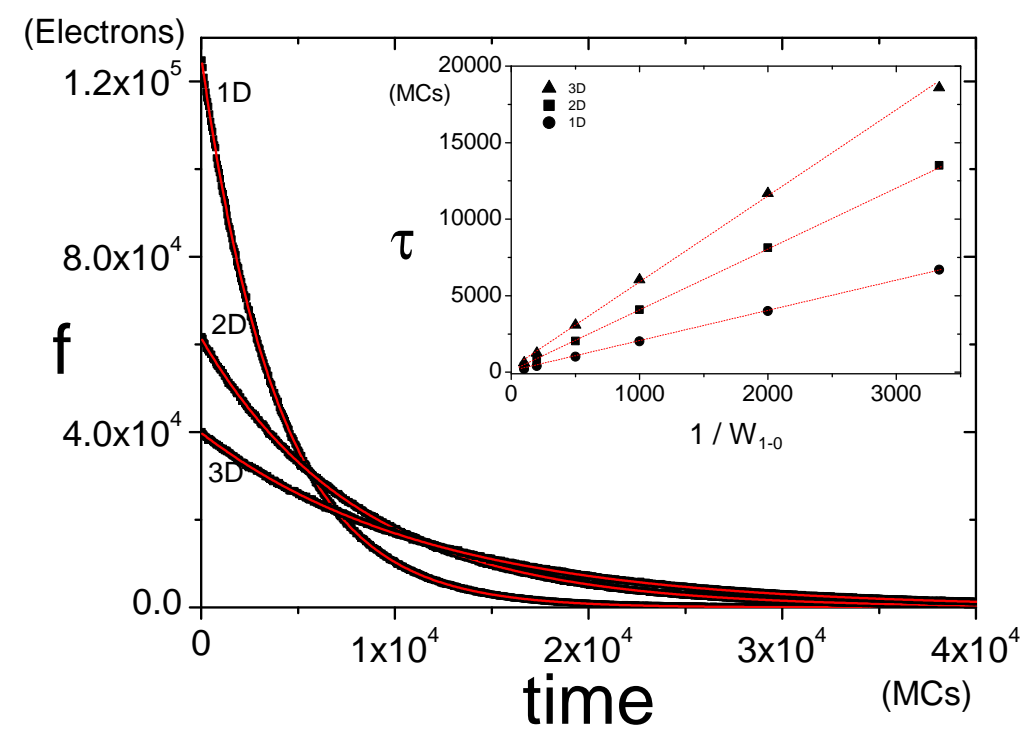

Figure 5: (color online)The distribution of electron lifetimes before recombining for $1 \mathrm{D}, 2 \mathrm{D}$ and $3 \mathrm{D}$ systems for $w_{10}=0.0005$. The exponential fits are given by the lines. In the insert: the relaxation times of the recombination times distributions versus the inverse recombination rates for the systems of different dimensionality; the lines are the results of linear fitting. 